\title{
The Prevalence of Dyslipidemia among Jordanians
}

\author{
Mousa Abujbara, ${ }^{1}$ Anwar Batieha $\left(\mathbb{D},{ }^{2}\right.$ Yousef Khader $\left(\mathbb{D},{ }^{3}\right.$ Hashem Jaddou, ${ }^{4}$ \\ Mohammed El-Khateeb, ${ }^{1}$ and Kamel Ajlouni ${ }^{1}{ }^{1}$ \\ ${ }^{1}$ The National Center (Institute) for Diabetes, Endocrinology and Genetics (NCDEG)/The University of Jordan, Jordan \\ ${ }^{2}$ Department of Community Medicine, Jordan University of Science and Technology, Irbid, Jordan \\ ${ }^{3}$ Jordan University of Science and Technology (JUST), Irbid, Jordan \\ ${ }^{4}$ The Jordan University of Science and Technology (JUST), Irbid, Jordan
}

Correspondence should be addressed to Kamel Ajlouni; ajlouni@ju.edu.jo

Received 5 September 2018; Accepted 23 October 2018; Published 28 October 2018

Academic Editor: Gerd Schmitz

Copyright (C) 2018 Mousa Abujbara et al. This is an open access article distributed under the Creative Commons Attribution License, which permits unrestricted use, distribution, and reproduction in any medium, provided the original work is properly cited.

\begin{abstract}
Background. Dyslipidemia is one of the major modifiable risk factors for the development of cardiovascular disease and type two diabetes mellitus. Knowing the current prevalence of dyslipidemia is an important step for increasing awareness of the problem and for proper planning of health programs for prevention of its negative clinical effects. Methods. A national population based household sample was selected from north, middle, and south regions of Jordan in 2017. A total sample of 4,056 aged between 18 and 90 years were included. Selected individuals were interviewed using a structured questionnaire and fasting blood samples were collected for biochemical measurements. Results. The prevalence rates of hypercholesterolemia, hypertriglyceridemia, high LDL, and low HDL were 44.3\%, 41.9\% 75.9\%, and 59.5\%, respectively. Hypercholesterolemia in Jordan almost doubled from $23.0 \%$ in 1994 to $44.3 \%$ in 2017, and hypertriglyceridemia increased from $23.8 \%$ in 1994 to $41.9 \%$ in 2017. All lipid abnormalities decreased after the age of 60 years. Hypertension, diabetes, and obesity were all independently associated with hypercholesterolemia and hypertriglyceridemia. Conclusions. Results of this study show that dyslipidemia is a widely prevalent health problem among adult Jordanian population and that the problem has substantially increased since 1994. Encouraging healthy lifestyle and healthy diet are the basis for prevention of dyslipidemia.
\end{abstract}

\section{Introduction}

Noncommunicable diseases (NCDs) are considered the leading cause of death in Jordan, with $38 \%$ of deaths attributed to cardiovascular diseases (CVDs) [1]. With the rapid lifestyle changes and socioeconomic development, the prevalence of NCDs has markedly increased over the past years in Jordan [2]. It was estimated that between 1 and 3 million persons in Jordan will have hypertension, high blood cholesterol, or diabetes by 2050 [3]. Dyslipidemia is one of the major modifiable risk factors for the development of cardiovascular diseases, atherosclerosis, and type two diabetes mellitus [4]. Guidelines for screening and treating dyslipidemia indicate that proper management of lipids is both lifesaving and costeffective. Furthermore, recent research shows that the association between blood cholesterol level and atherosclerotic cardiovascular diseases is independent of other risk factors and causal associations [5]. Knowing the current prevalence of dyslipidemia is an important step for increasing awareness of the problem and for proper planning of health programs for prevention of the problem and its negative clinical effects. In 1994, a national community-based survey was conducted to estimate the prevalence of dyslipidemia and other cardiovascular risk factors in Jordan [6]. The present study is a follow-up survey using generally a similar methodology, to assess the current prevalence of dyslipidemia in Jordan and to identify its distribution among subgroups of the population.

\section{Materials and Methods}

A cross-sectional community-based survey targeting adults, $\geq 18$ years of age in 2017, was conducted by the National Center for Diabetes, Endocrinology and Genetics (NCDEG), Jordan, Ministry of Health $(\mathrm{MoH})$, and Jordan University of Science 
and Technology (JUST). A multistage sampling technique was used to represent rural and urban population in the three geographic regions of Jordan (north, middle, and south). As the survey procedures have to take place in health facilities (anthropometric measures, drawing blood samples...etc.). General Health Directors of each of the 12 governorates of Jordan were asked to select one to three health centers that could represent the rest of health centers in each governorate. The selected health centers had to be large enough to provide the needed space for the survey team ( 25 persons) to perform the required procedures. A team of two field researchers (a male and a female) went door to door to invite a systematic sample of households in the catchment area of the selected center.

The team explained the study protocol and responded to all questions. If they agreed to participate, they were asked to report to the health center at 8:00 am, fasting for ten hours, and to bring all of their medications with them to be checked by the survey team. They were also asked not to take their medications on the day of their visit to the health center. All household members $\geq 18$ years of age were eligible to participate in the study. However, only subjects $\geq 25$ years of age were included in this analysis. The reason for including only this age group in the analysis is to allow for comparison with the previous national survey and with results from other countries. To encourage participation in the study, visit dates to health centers were negotiated with eligible participants. The study team worked all weekdays (except Fridays) to obtain the highest possible response rate. If a person refused to participate, basic data were collected when possible such as age, gender, education, whether the person was diagnosed with any NCDs, and the reason for nonparticipation. Total response rate was $40 \%$ for males and $94 \%$ for females. A total sample of 4,056 subjects completed the survey and were included in the analysis.

The survey protocol was approved by the Ethics Committee of the National Center for Diabetes, Endocrinology, and Genetics, Amman, Jordan. A letter of support was obtained from the Minister of Health $(\mathrm{MoH})$ to ensure the cooperation of the staff of the health centers. An informed consent was obtained from all study participants. Study participants were allowed to decline participation in any part of the study. All study procedures were carried out free of charge for the participants. Data were treated with high confidentiality and used only for scientific purposes.

All field researchers attended a two-day training workshop, prior to data collection. Training included explaining the study purpose and procedures, proper interview skills, and standard methods of anthropometric measurements. Health workers responsible for obtaining blood samples were trained on the standard aseptic technique for obtaining blood samples.

Selected individuals were interviewed using a structured questionnaire that inquired about socio-demographic characteristics, smoking and other variables related to this study. Height was measured without footwear using a portable stadiometer and the reading was recorded to the nearest 1 $\mathrm{cm}$. Weight was measured by a standard weighing scale in light clothes and without footwear with a precision of $100 \mathrm{gm}$.
Body mass index (BMI) was calculated as weight divided by height squared $\left(\mathrm{kg} / \mathrm{m}^{2}\right)$. Waist circumference was measured by a nonstretchable tape at the narrowest horizontal plane midway between the iliac crest and lower costal margin with a $1 \mathrm{~cm}$ precision. Hip circumference was measured at the widest horizontal plane at the hip level with a precision of $1 \mathrm{~cm}$. Waist to height ratio was calculated as waist circumference divided by height. Three blood samples were drawn from a cannula inserted into the antecubital vein using vacutainer tubes. Collected blood samples were centrifuged within one hour in the field and transported on daily basis in cold boxes from the field to the Central Laboratory of the National Center for Diabetes, Endocrinology, and Genetics in Amman, Jordan, where all laboratory measurements were performed. All biochemical measurements were done by the same team of laboratory technicians using the same method throughout the survey period. Lipids measurements were performed according to the manufacturers' instructions, using COBAS autoanalyzer (Roche Diagnostics, Basel, Switzerland).

\section{Definitions of Variables}

Lipid variables were defined according to the Third Report of the National Cholesterol Education Program (NCEP-Adult Treatment Panel III). Hypercholesterolemia was defined as a serum cholesterol level of $\geq 5.17 \mathrm{mmol} / \mathrm{l}(200 \mathrm{mg} / \mathrm{dl})$ or on lipid-lowering medication; high low-density lipoprotein (LDL-C) was defined as LDL-C level of $\geq 2.59 \mathrm{mmol} / \mathrm{l}$ (100 $\mathrm{mg} / \mathrm{dl}$ ) or on lipid-lowering medication; low high-density lipoprotein (HDL-C) was defined as HDL-C level of $\leq 1.03$ $\mathrm{mmol} / \mathrm{l}(40 \mathrm{mg} / \mathrm{dl})$ in men, and $\leq 1.29 \mathrm{mmol} / \mathrm{l}(50 \mathrm{mg} / \mathrm{dl})$ in women or on lipid-lowering medication. Hypertriglyceridemia was defined as a serum triglyceride (TG) $\geq 1.70$ $\mathrm{mmoi} / \mathrm{l}(150 \mathrm{mg} / \mathrm{dl})$ or on lipid-lowering medication. Total cholesterol to HDL-C ratio (TC/HDL ratio) was considered high if the ratio was $\geq 5$. Hypertension was defined as systolic blood pressure $\geq 130 \mathrm{mmHg}$ or diastolic blood pressure $>85$ $\mathrm{mmHg}$ or if the patient was on antihypertensive drugs. Diabetes mellitus was defined as a fasting plasma glucose $\geq$ $7.0 \mathrm{mmol} / \mathrm{l}(126 \mathrm{mg} / \mathrm{dl})$ or a $\mathrm{HbAlC} \geq 6.5 \%$, or on treatment for diabetes. Smoking was classified into nonsmoker and current smoker (smoked cigarettes daily or occasionally). Participants with BMI between 25 and 29.9 were considered overweight and with BMI of 30 or more were considered obese. Waist circumference was considered abnormal at $\geq 94$ $\mathrm{cm}$ in men and $\geq 80$ in women. Waist-to-height ratio was considered elevated if it was $>0.5$.

\section{Statistical Analysis}

Survey data were analyzed using the Statistical Package for Social Sciences (SPSS Version 23). Range and logical checks were used to detect errors in data entry. Detected errors were corrected by returning to the original data forms. A P-value of $\leq 0.05$ was considered statistically significant. Comparisons were made between participants with dyslipidemia and the rest of the sample to identify potential associations that were tested for statistical significance using the chi square test for categorical variables. Multivariate logistic regression 
was used to determine the effect of a given variable while simultaneously controlling for potential confounders. The magnitude of the associations was assessed by the adjusted odds ratio, and the statistical significance was assessed by the Wald chi square.

\section{Results}

This study included 4,056 subjects (70.6\% females and 29.4\% males) aged between 18 and 90 years with a mean age (SD) of 43.7 (14.2) years. The majority of the subjects were either overweight or obese $(74.5 \%)$ and $14.5 \%$ of them were current smokers. The prevalence rates of hypercholesterolemia, hypertriglyceridemia, high LDL and low HDL were $44.3 \%$, $41.9 \% 75.9 \%$, and $59.5 \%$, respectively.

Table 1 shows the prevalence of dyslipidemia according to subjects' socio-demographic characteristics. Prevalence of hypercholesterolemia $(\geq 5.17 \mathrm{mmol} / \mathrm{l})$ in females was $44.8 \%$ and $43.0 \%$ in males. Hypertriglyceridemia $(\geq 1.70 \mathrm{mmol} / \mathrm{l})$ was $36.5 \%$ in females significantly lower than in males (54.6\%). Study participants showed no statistical difference in prevalence of low HDL-C and high LDL-C between male and female participates. On the other hand, the prevalence of TC/HDL ratio $>5$ in males was double that in females and the difference was statistically significant. The prevalence of hypercholesterolemia and hypertriglyceridemia reached its peak at the age group of 50-59 years then decreased again, while the prevalence of low HDL-C increased until the age of 39 years and it plateaued at 40 to 69 years to decrease at age 70. The prevalence of low LDL-C reached its peak between 40 to 49 years then decreased gradually among the older age groups. Similar significant change in the prevalence of TC/HDL ratio $>5$ was noticed where the prevalence increased until the age group of 50 to 59 years then decreased in the older age groups. Prevalence of hypercholesterolemia and hypertriglyceridemia was significantly higher among obese and overweight participants than normal BMI participants where the prevalence of hypercholesterolemia and TG was $50.6 \%$ and $52.7 \%$, respectively, in obese participants. Also, the highest prevalence of low HDL-C and high LDL-C and TC/HDL ratio $>5$ was noticed among obese participants. Likewise, participants with waist-to-height ratio $>0.5$ had hypercholesterolemia, hypertriglyceridemia, low HDLC, high LDL-C, and TC/HDL ratio $>5$ prevalence significantly higher than participants with waist-to-height ratio $\leq$ 0.5 . The difference in prevalence of dyslipidemias was not statistically significant in different regions in Jordan except for hypertriglyceridemia which was lower in participants living in the middle region in comparison with participants living in other regions. Study results showed no significant difference in hypercholesterolemia between smokers and nonsmokers, while the prevalence of hypertriglyceridemia in smokers (53.9\%) was significantly higher than in nonsmokers (39.7\%). Low HDL-C was significantly higher in smokers than nonsmokers, while high LDL-C was not significantly different between smokers and nonsmokers. TC/HDL ratio $>5$ was significantly more prevalent in smokers than in nonsmokers. On bivariate analysis, the prevalence of hypercholesterolemia and high LDL-C was almost the same in diabetic and nondiabetic subjects and in hypertensive and normotensive subjects. While, hypertriglyceridemia, low HDL-C, and TC/HDL ratio $>5$ prevalence were all significantly higher in diabetic subjects when compared with nondiabetics and in hypertensive subjects when compared with normotensive subjects.

Tables 2-5 shows multivariate regression findings for hypercholesterolemia, hypertriglyceridemia, low HDL-C, and high LDL-C, respectively. Table 2 shows that hypercholesterolemia was significantly associated with hypertension (Odds Ratio $(\mathrm{OR})=1.37$, 95\% Confidence Interval $(\mathrm{CI})=$ 1.15-1.63) and diabetes ( $\mathrm{OR}=1.25,95 \% \mathrm{CI}=1.04-1.50)$. Age, overweight, and obesity were all significantly associated with increased risk of hypercholesterolemia. Table 3 shows that hypertriglyceridemia was significantly associated with hypertension $(\mathrm{OR}=1.36,95 \% \mathrm{CI}=1.13-1.62)$, diabetes $(\mathrm{OR}=1.68,95 \%$ $\mathrm{CI}=1.39-2.04)$, and cigarette smoking $(\mathrm{OR}=1.53,95 \% \mathrm{CI}=1.24$ 1.89). Similarly, overweight subjects were more likely to have hypertriglyceridemia $(\mathrm{OR}=2.01,95 \% \mathrm{CI}=1.61-2.50)$ and obese subjects were also more likely to have hypertriglyceridemia $(\mathrm{OR}=2.89,95 \% \mathrm{CI}=2.33-3.58)$ compared with subjects with normal BMI. Study subjects in 40-49, 50-59, and 60-69 age groups were more likely to have hypertriglyceridemia compared to subjects less than 40 years old, whereas female gender $(\mathrm{OR}=0.49,95 \% \mathrm{CI}=0.42-0.57)$, being single and living in the middle or south region were all independent protective factors from hypertriglyceridemia. Table 4 shows that low HDL-C was significantly associated with hypertension $(\mathrm{OR}=1.31,95 \% \mathrm{CI}=1.10-1.55)$, diabetes $(\mathrm{OR}=1.47$, $95 \% \mathrm{CI}=1.21-1.77$ ), waist circumference $>94 \mathrm{~cm}$ in men or $>80 \mathrm{~cm}$ in women $(\mathrm{OR}=1.48,95 \% \mathrm{CI}=1.21-1.81)$, and obesity $(\mathrm{OR}=1.75,95 \% \mathrm{CI}=1.41-2.22)$, while female gender was an independent protective factor from low HDL-C (OR $=0.83$, $95 \% \mathrm{CI}=0.71-0.96)$. Table 5 shows that high LDL-C was significantly associated with hypertension $(\mathrm{OR}=1.35,95 \%$ $\mathrm{CI}=1.09-1.67)$, diabetes $(\mathrm{OR}=1.66,95 \% \mathrm{CI}=1.33-2.07)$, and abnormal waist circumference (OR=1.63, 95\% $\mathrm{CI}=1.35-1.96)$. In addition, 40-49-, 50-59-, 60-69-, and a 70-year age groups were significantly more likely to have high LDL-C compared to subjects less than 40 years old. Also, overweight and obese subjects were more likely to have high LDL-C compared to subjects with normal BMI.

\section{Discussion}

Results of this study show that dyslipidemia is a widely prevalent health problem affecting adult Jordanian population and putting them at an enormous risk for CVD $[7,8]$. To the best of our knowledge, this study is one of a few community-based studies conducted in Jordan over the past 23 years. The prevalence of hypercholesterolemia in Jordan increased from $23.0 \%$ in 1994 to $44.3 \%$ in 2017, and the prevalence of hypertriglyceridemia increased from $23.8 \%$ in 1994 to $41.9 \%$ in 2017 [6]. This marked increase could be explained by lifestyle changes and by changes in food consumption pattern. However, better survival of subjects with dyslipidemia may have contributed to this increase.

A remarkable finding of this study was that all types of lipid abnormalities decrease after the age of 60 years. Streja et 
TABLE 1: Prevalence of dyslipidemia by selected variables, Jordan, 2017.

\begin{tabular}{|c|c|c|c|c|c|}
\hline Variables & $\begin{array}{c}\text { Hypercholesterolemia } \\
\geq 5.17 \mathrm{mmol} / \mathrm{l} \\
\mathrm{n}(\%)\end{array}$ & $\begin{array}{c}\text { Hypertriglyceridemia } \\
\geq 1.70 \mathrm{mmol} / \mathrm{l} \\
\mathrm{n}(\%)\end{array}$ & $\begin{array}{c}\text { Low HDL-C } \\
\mathrm{F} \leq 1.29 \mathrm{mmol}, \mathrm{M} \\
\leq 1.03 \mathrm{mmol} / \mathrm{l} \\
\mathrm{n}(\%)\end{array}$ & $\begin{array}{l}\text { High LDL-C } \\
\geq 2.59 \mathrm{mmol} / 1 \\
\mathrm{n}(\%)\end{array}$ & $\begin{array}{c}\text { TC/HDL ratio } \\
>5 \\
\mathrm{n}(\%)\end{array}$ \\
\hline Overall & $1780(44.3)$ & $1683(41.9)$ & $2391(59.5)$ & 3049 (75.9) & $1335(33.2)$ \\
\hline \multicolumn{6}{|l|}{ Gender } \\
\hline Male & $509(43.0)$ & $647(54.6)$ & $732(61.8)$ & $883(74.6)$ & $619(52.3)$ \\
\hline Female & $1271(44.8)$ & $1036(36.5)$ & $1659(58.5)$ & $2166(76.4)$ & $716(25.3)$ \\
\hline P-value & 0.274 & 0.000 & 0.052 & 0.218 & 0.000 \\
\hline \multicolumn{6}{|l|}{ Age (years) } \\
\hline $25-29$ & $83(29.5)$ & $58(20.6)$ & $120(42.7)$ & $179(63.7)$ & $44(15.7)$ \\
\hline $30-39$ & $324(40.5)$ & $281(35.1)$ & $449(56.1)$ & $611(76.4)$ & $219(27.4)$ \\
\hline $40-49$ & $562(52.7)$ & $516(48.4)$ & $665(62.4)$ & $907(85.2)$ & $419(39.3)$ \\
\hline $50-59$ & $467(55.7)$ & $457(54.5)$ & $550(65.6)$ & $696(83.1)$ & $377(45.0)$ \\
\hline $60-69$ & $208(51.9)$ & $245(61.1)$ & $269(67.1)$ & $314(78.3)$ & $178(44.4)$ \\
\hline$\geq 70$ & 75 (41.9) & $78(43.6)$ & $104(58.1)$ & $132(73.7)$ & $62(34.6)$ \\
\hline $\mathrm{P}$-value & 0.000 & 0.000 & 0.000 & 0.000 & 0.000 \\
\hline \multicolumn{6}{|l|}{ BMI $\left(\mathrm{kg} / \mathrm{m}^{2}\right)$} \\
\hline Normal & $249(31.0)$ & $166(20.7)$ & $364(45.3)$ & $499(62.1)$ & $142(17.7)$ \\
\hline Overweight & $602(46.4)$ & $548(42.3)$ & $735(56.7)$ & $1030(79.4)$ & $446(34.4)$ \\
\hline Obese & $916(50.6)$ & $954(52.7)$ & $1243(68.7)$ & $1467(81.1)$ & $737(40.7)$ \\
\hline P-value & 0.000 & 0.000 & 0.000 & 0.000 & 0.000 \\
\hline \multicolumn{6}{|l|}{$\begin{array}{l}\text { Waist to height } \\
\text { ratio }\end{array}$} \\
\hline Normal & $954(40.2)$ & $787(33.1)$ & $1246(52.5)$ & $1714(72.2)$ & $620(26.1)$ \\
\hline Abnormal & $821(50.4)$ & $890(54.6)$ & $1135(69.7)$ & $1323(81.2)$ & $711(43.6)$ \\
\hline P-value & 0.000 & 0.000 & 0.000 & 0.000 & 0.000 \\
\hline \multicolumn{6}{|l|}{$\begin{array}{l}\text { Geographical } \\
\text { region }\end{array}$} \\
\hline North & $579(44.7)$ & $602(46.5)$ & $785(60.6)$ & $991(76.5)$ & $441(34.1)$ \\
\hline Middle & $784(44.4)$ & $648(36.7)$ & $1030(58.4)$ & $1316(74.6)$ & $559(31.7)$ \\
\hline South & $417(43.4)$ & $433(45.1)$ & $576(60.0)$ & $742(77.3)$ & $335(34.9)$ \\
\hline P-value & 0.809 & 0.000 & 0.433 & 0.251 & 0.175 \\
\hline \multicolumn{6}{|l|}{ Marital status } \\
\hline Married & $1452(47.2)$ & $1422(46.2)$ & $187(61.0)$ & $2438(79.2)$ & $1131(36.8)$ \\
\hline Single & $141(23.7)$ & $88(14.8)$ & $298(50.0)$ & $331(55.5)$ & $74(12.4)$ \\
\hline Divorced or widow & $180(53.9)$ & $164(49.1)$ & $206(61.7)$ & $271(81.1)$ & $122(36.5)$ \\
\hline P-value & 0.000 & 0.000 & 0.000 & 0.000 & 0.000 \\
\hline \multicolumn{6}{|l|}{ Smoking } \\
\hline Smoker & $258(44.4)$ & $313(53.9)$ & $420(72.4)$ & $444(76.6)$ & $316(54.5)$ \\
\hline Non-smoker & $1513(44.3)$ & $1356(39.7)$ & $1957(57.2)$ & $2590(75.8)$ & $1009(29.5)$ \\
\hline P-value & 0.945 & 0.000 & 0.000 & 0.678 & 0.000 \\
\hline \multicolumn{6}{|l|}{ Hypertension } \\
\hline Yes & $438(46.3)$ & $563(59.5)$ & $664(70.2)$ & $729(77.1)$ & $414(43.8)$ \\
\hline No & $1329(43.7)$ & $1103(36.3)$ & $1704(56.0)$ & $2296(75.5)$ & $908(29.9)$ \\
\hline P-value & 0.160 & 0.000 & 0.000 & 0.000 & 0.000 \\
\hline \multicolumn{6}{|l|}{ Diabetes mellitus } \\
\hline Yes & $353(46.3)$ & $485(63.6)$ & $547(71.9)$ & $564(74.1)$ & $341(44.8)$ \\
\hline No & $1420(43.8)$ & $1186(36.6)$ & $1832(56.5)$ & $2471(76.3)$ & $985(30.4)$ \\
\hline P-value & 0.212 & 0.000 & 0.000 & 0.212 & 0.000 \\
\hline
\end{tabular}


TABle 2: Adjusted odds ratios and their 95\% $\mathrm{Cl}$ for Hypercholesterolemia using multivariate logistic regression, Jordan 2017.

\begin{tabular}{lcc}
\hline Variable & Adjusted OR & 95\% CI \\
\hline Gender & 1 & \\
Male & 1.17 & $1.00-1.35$ \\
Female & 1 & \\
\hline Age (years) & 2.37 & $1.99-2.82$ \\
$<40$ & 2.87 & $2.36-3.49$ \\
$40-49$ & 2.73 & $2.11-3.53$ \\
$50-59$ & 1.92 & $3.62-6.67$ \\
$60-69$ & & \\
$\geq 70$ & 1 & $1.34-1.97$ \\
\hline BMI & 1.63 & $1.46-2.14$ \\
Normal & 1.77 & \\
Overweight & & $1.15-1.63$ \\
Obese & 1 & \\
\hline Hypertension & 1.37 & $1.04-1.50$ \\
No & & \\
Yes & 1 & \\
\hline Diabetes & 1.25 & \\
No & & \\
Yes & &
\end{tabular}

al. reported similar findings in a chapter about management of dyslipidemia in the elderly [9]. A possible explanation for this finding is that many people with dyslipidemia might die before the age of 60 , leaving only a few surviving to this age. Attrition or survival bias can decrease associations between harmful exposures and illnesses of aging [9].

Previous studies from our region showed variable results. In a study of Kuwaiti adults, $33.7 \%$ of men and $30.6 \%$ of women were reported to have hypercholesterolemia. Another study in Oman reported hypercholesterolemia prevalence of $33.6 \%[10,11]$. On the other hand, a higher prevalence of hypercholesterolemia was reported from Saudi Arabia (54\%) [12] while an Iranian meta-analysis reported a hypercholesterolemia prevalence of $41.6 \%$ which is very close to our figure of $44.3 \%$ [13]. Differences in study design, dietary habits, lifestyles, and accessibility to health care may account for much of the variations in the prevalence of hypercholesterolemia.

Hypertriglyceridemia prevalence in the current study population (41.9\%) was close to that reported from Saudi Arabia (40.3\%) [12], and Iran (46.0\%) [13].

The present study showed the independent risk factors of the different types of dyslipidemia by multivariate analysis. Diabetes mellitus and hypertension were independent risk factors for hypercholesterolemia. A study from Saudi Arabia also reported same association between diabetes mellitus, hypertension, and hypercholesterolemia [14]. Similarly, obesity was an independent risk factor for hypercholesterolemia in the current study, in an Ethiopian national survey and in a study from Beijing China $[15,16]$. Additionally, hypertriglyceridemia was independently associated with diabetes
TABle 3: Adjusted odds ratios and their $95 \% \mathrm{Cl}$ for Hypertriglyceridemiausing multivariate logistic regression, Jordan, 2017.

\begin{tabular}{|c|c|c|}
\hline Variable & Adjusted OR & 95\% CI \\
\hline \multicolumn{3}{|l|}{ Gender } \\
\hline Male & 1 & \\
\hline Female & 0.49 & $0.42-0.57$ \\
\hline \multicolumn{3}{|l|}{ Age (years) } \\
\hline$<40$ & 1 & \\
\hline $40-49$ & 1.65 & $1.37-1.98$ \\
\hline $50-59$ & 1.64 & $1.33-2.02$ \\
\hline $60-69$ & 1.84 & $1.40-2.42$ \\
\hline$\geq 70$ & 0.44 & $0.60-1.24$ \\
\hline \multicolumn{3}{|l|}{ BMI } \\
\hline Normal & 1 & \\
\hline Overweight & 2.01 & $1.61-2.50$ \\
\hline Obese & 2.89 & $2.33-3.58$ \\
\hline \multicolumn{3}{|l|}{ Hypertension } \\
\hline No & 1 & \\
\hline Yes & 1.36 & $1.13-1.62$ \\
\hline \multicolumn{3}{|l|}{ Diabetes } \\
\hline No & 1 & \\
\hline Yes & 1.68 & $1.39-2.04$ \\
\hline \multicolumn{3}{|l|}{ Marital status } \\
\hline Married & 1 & \\
\hline Single & 0.41 & $0.31-0.53$ \\
\hline Divorced or widow & 1.06 & $0.82-1.37$ \\
\hline \multicolumn{3}{|l|}{ Geographical region } \\
\hline North & 1 & \\
\hline Middle & 0.68 & $0.58-0.80$ \\
\hline South & 0.77 & 0.64-0.93 \\
\hline \multicolumn{3}{|l|}{ Smoking } \\
\hline Non-smoker & 1 & \\
\hline Smoker & 1.53 & $1.24-1.89$ \\
\hline
\end{tabular}

mellitus. A similar association between hypertriglyceridemia and $\mathrm{HbAlc}$ was reported in a Russian study [17].

An interesting finding was that living in northern region of Jordan was an independent risk factor for hypertriglyceridemia. We have no clear explanation for this finding which may need further investigation.

The prevalence of low HDL-C and prevalence of high LDL-C were positively and independently associated with obesity, increased waist circumference, diabetes, and hypertension. This is consistent with a recent study in Turkey [18]. There have been a lot of changes regarding the optimal management of dyslipidemia in the last few years. The American Association of Clinical Endocrinologists and American College of Endocrinology 2017 guidelines (AACE 2017) for management of dyslipidemia and prevention of cardiovascular disease encourage physicians to provide personalized goals of lipid levels to their patients and states that the 10year risk of a coronary event need to be assessed in order to provide such personalized lipid management [5]. 
TABLE 4: Adjusted odds ratios and their 95\% Cl for low HDL-C using multivariate logistic regression, Jordan 2017.

\begin{tabular}{lcc}
\hline Variable & Adjusted OR & 95\% CI \\
\hline Gender & 1 & \\
Male & 0.83 & $0.71-0.96$ \\
Female & 1 & \\
\hline BMI & 1.18 & $0.96-1.46$ \\
Normal & 1.75 & $1.41-2.22$ \\
Overweight & & \\
Obese & 1 & $1.21-1.81$ \\
\hline Waist circumference & 1.48 & \\
Normal & & \\
Increased & 1 & \\
\hline Hypertension & 1.31 & $1.10-1.55$ \\
No & & \\
Yes & 1 & \\
\hline Diabetes & 1.47 & \\
No & & \\
Yes & &
\end{tabular}

TABLE 5: Adjusted odds ratios and their $95 \% \mathrm{Cl}$ for high LDL-Cusing multivariate logistic regression, Jordan 2017.

\begin{tabular}{lcc}
\hline Variable & Adjusted OR & 95\% CI \\
\hline Gender & 1 & \\
Male & 1.19 & $1.00-1.43$ \\
Female & & \\
\hline Age (years) & 1 & \\
$<40$ & 2.90 & $2.33-3.60$ \\
$40-49$ & 2.76 & $2.16-3.52$ \\
$50-59$ & 2.29 & $1.68-3.12$ \\
$60-69$ & 1.95 & $1.32-2.87$ \\
$\geq 70$ & & \\
\hline BMI & 1 & $1.69-2.54$ \\
Normal & 2.07 & $1.73-2.58$ \\
Overweight & 2.11 & \\
Obese & & \\
\hline Waist circumference & 1 & $1.35-1.96$ \\
Normal & 1.63 & \\
Increased & & \\
\hline Hypertension & 1 & \\
No & 1.35 & \\
Yes & $1.69-1.67$ \\
\hline Diabetes & & \\
No & & \\
Yes & & \\
\hline
\end{tabular}

This study had two main limitations: firstly, it was a crosssectional survey, in which the risk factors and dyslipidemia were assessed simultaneously. Consequently, it is not possible to judge whether dyslipidemia was present before or after the proposed risk factors; i.e., the temporal sequence from cause to effect could not be established. Secondly, our associations are based on prevalence rather incidence. Prevalence is affected by duration of the disease which may in turn be related to health care; thus differences in prevalence may merely reflect differences in survival rather differences in incidence.

In conclusion, it was clear that dyslipidemia is high in Jordan which may put a high burden on the already strained health services and leads to substantial increase in health related costs. Prevention of dyslipidemia is critical to achieve the goal of halting the rise in cardiovascular diseases. At the community level, encouraging healthy lifestyle, healthy dietary habits, and physical exercise are the cornerstone for prevention of dyslipidemia. Screening of high risk groups such as obese people is recommended.

\section{Data Availability}

The SPSS data used to support the findings of this study are available from the corresponding author upon request.

\section{Conflicts of Interest}

The authors declare that there are no conflicts of interest regarding the publication of this paper."

\section{Authors' Contributions}

Mousa Abujbara wrote the manuscript, approved the protocol, analyzed the data, and approved the results. Anwar Batieha is the main author of the study protocol and a Professor of epidemiology and public health and he is responsible for the statistical part of the study. Yousef Khader shared with Dr. Batieha the responsibility for statistics and supervised the fieldwork. Hashem Jaddou is the co-author of the structured questionnaire and supervised the execution of the protocol and data collection. Mohammed El-Khateeb is responsible for all the lab procedures, samples handling, transport, and analysis. Kamel Ajlouni is the Chairman of the National Strategic Committee, the main investigator, the principle author of the study, and the guarantor, who shared in planning executing and shared with the analysis of the data.

\section{References}

[1] A. Mokdad, "Global non-communicable disease prevention: Building on success by addressing an emerging health need in developing countries," Journal of Health Specialties, vol. 4, no. 2, p. 92, 2016

[2] Ministry of Health, The national strategy and plan of action against diabetes, hypertension, dyslipidemia and obesity in Jordan, 2011, http://www.moh.gov.jo.

[3] D. W. Brown, A. H. Mokdad, H. Walke et al., "Projected burden of chronic, noncommunicable diseases in Jordan," Preventing Chronic Disease, vol. 6, no. 2, article no. A78, 2009.

[4] L. Qi, X. Ding, W. Tang, Q. Li, D. Mao, and Y. Wang, "Prevalence and risk factors associated with dyslipidemia in Chongqing, China," International Journal of Environmental Research and Public Health, vol. 12, no. 10, pp. 13455-13465, 2015. 
[5] P. S. Jellinger, Y. Handelsman, P. D. Rosenblit et al., "American Association of Clinical Endocrinologists and American College of Endocrinology guidelines for management of dyslipidemia and prevention of cardiovascular disease," Endocrine Practice, vol. 23, no. 4, pp. 1-87, 2017.

[6] A. Batieha, H. Y. Jaddou, and K. M. Ajlouni, "Hyperlipidemia in Jordan: A community-based survey," Saudi Medical Journal, vol. 18, no. 3, pp. 279-285, 1997.

[7] R. H. Nelson, "Hyperlipidemia as a Risk Factor for Cardiovascular Disease," Primary Care: Clinics in Office Practice, vol. 40, no. 1, pp. 195-211, 2013.

[8] H. O. Steinberg, B. Bayazeed, G. Hook, A. Johnson, J. Cronin, and A. D. Baron, "Endothelial dysfunction is associated with cholesterol levels in the high normal range in humans," Circulation, vol. 96, no. 10, pp. 3287-3293, 1997.

[9] D. Streja and E. Streja, Management of Dyslipidemia in the Elderly, 2014

[10] F. Ahmed, C. Waslien, M. Al-Sumaie, and P. Prakash, “Trends and risk factors of hypercholesterolemia among Kuwaiti adults: National Nutrition Surveillance Data from 1998 to 2009," Nutrition Journal, vol. 28, no. 9, pp. 917-923, 2012.

[11] A. Al Riyami, M. A. Abd Elaty, M. Morsi, H. Al Kharusi, W. Al Shukaily, and S. Jaju, "Oman World Health Survey: part 1-methodology, sociodemographic profile and epidemiology of non-communicable diseases in Oman," Oman Medical Journal, vol. 27, no. 5, pp. 425-443, 2012.

[12] M. M. Al-Nozha, M. R. Arafah, M. A. Al-Maatouq et al., "Hyperlipidemia in Saudi Arabia," Saudi Medical Journal, vol. 29, no. 2, pp. 282-287, 2008.

[13] A. Mohammadbeigi, E. Moshiri, N. Mohammadsalehi, H. Ansari, and A. Ahmadi, "Dyslipidemia Prevalence in Iranian Adult Men: The Impact of Population-Based Screening on the Detection of Undiagnosed Patients," The World Journal of Men's Health, vol. 33, no. 3, p. 167, 2015.

[14] M. Basulaiman, C. El Bcheraoui, M. Tuffaha et al., "Hypercholesterolemia and its associated risk factors-Kingdom of Saudi Arabia, 2013," Annals of Epidemiology, vol. 24, no. 11, pp. 801808, 2014.

[15] Y. F. Gebreyes, D. Y. Goshu, T. K. Geletew et al., "Prevalence of high bloodpressure, hyperglycemia, dyslipidemia, metabolic syndrome and their determinants in Ethiopia: Evidences from the National NCDs STEPS Survey, 2015," PLoS ONE, vol. 13, no. 5, p. e0194819, 2018.

[16] B. Jiang, A. J. Ma, H. Li et al., "Prevalence of hypercholesterolemia and influence factors in residents aged 18-65 years in Beijing," Zhonghua liu xing bing xue za zhi= Zhonghua liuxingbingxue zazhi, vol. 38, no. 7, pp. 938-943, 2017.

[17] Y. Karpov and Y. Khomitskaya, "PROMETHEUS: An observational, cross-sectional, retrospective study of hypertriglyceridemia in Russia," Cardiovascular Diabetology, vol. 14, no. 1, 2015.

[18] F. Bayram, D. Kocer, K. Gundogan et al., "Prevalence of dyslipidemia and associated risk factors in Turkish adults," Journal of Clinical Lipidology, vol. 8, no. 2, pp. 206-216, 2014. 


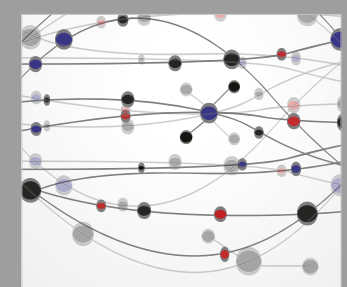

The Scientific World Journal
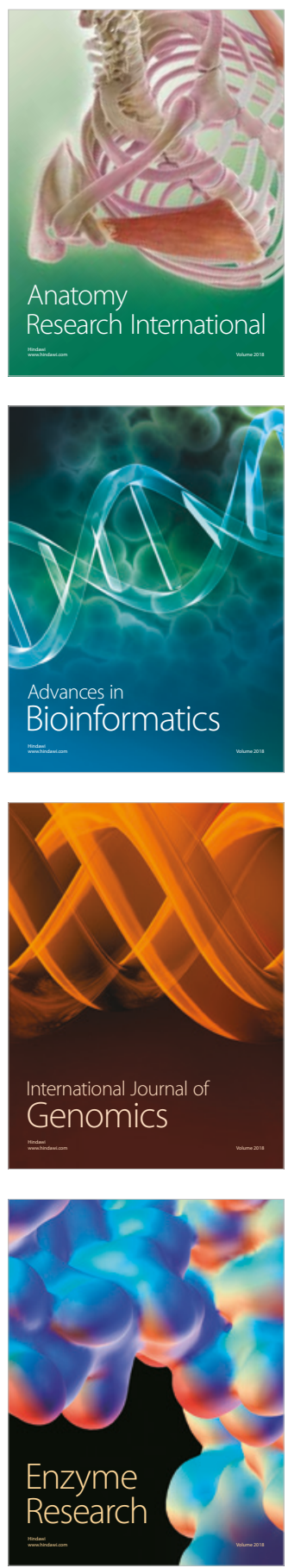
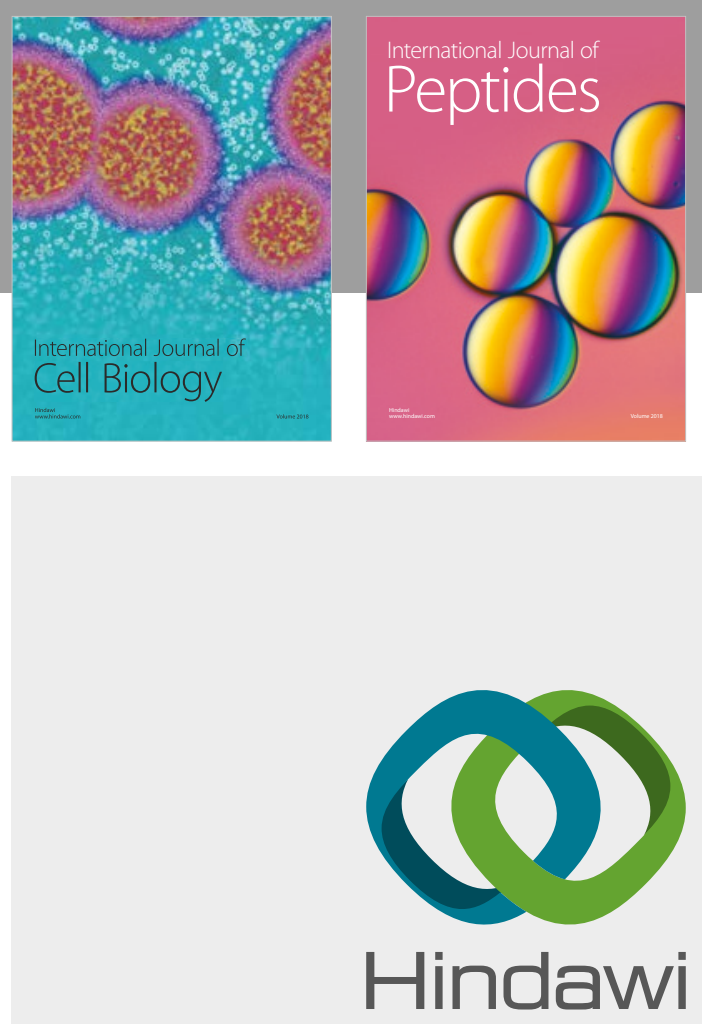

Submit your manuscripts at

www.hindawi.com
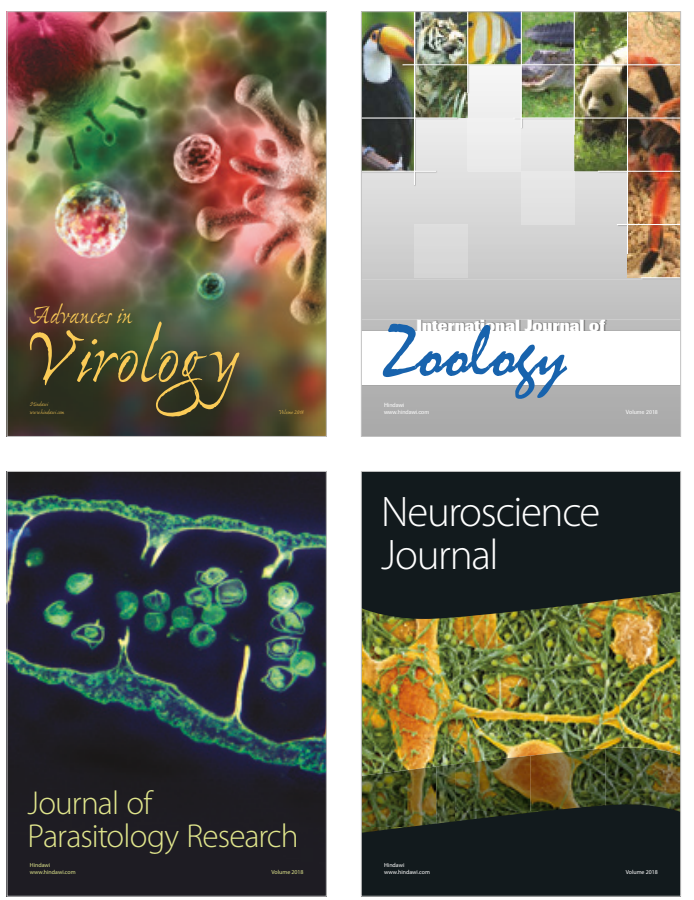
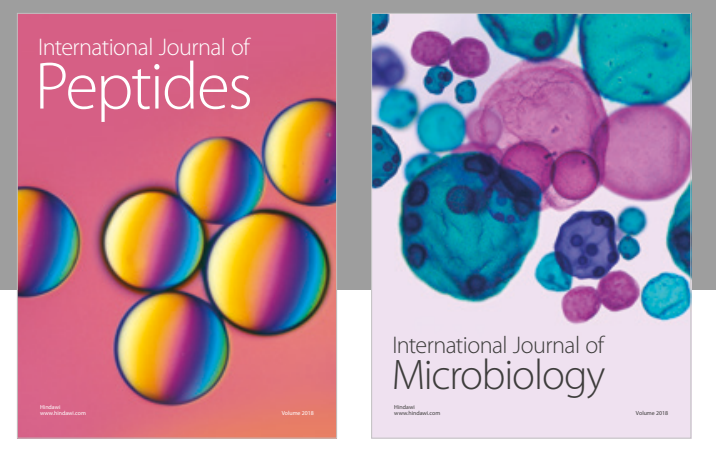

nternational Journal of Microbiology
Journal of
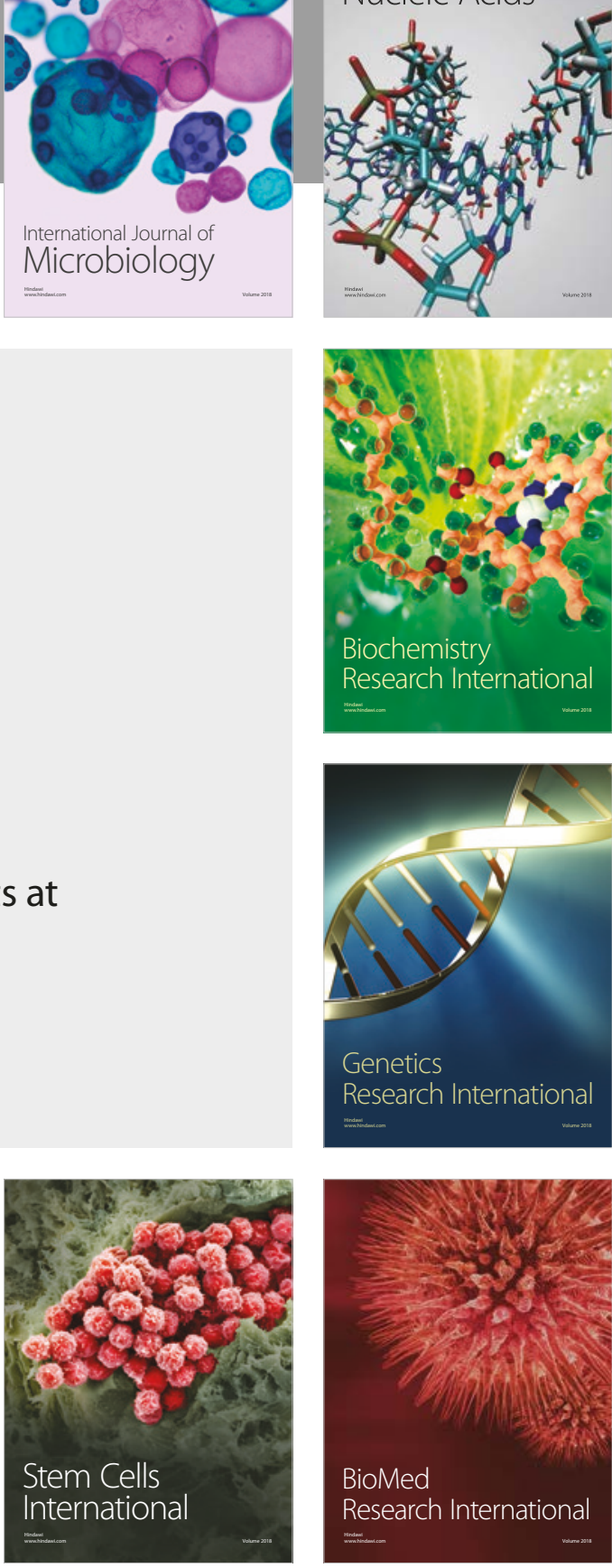
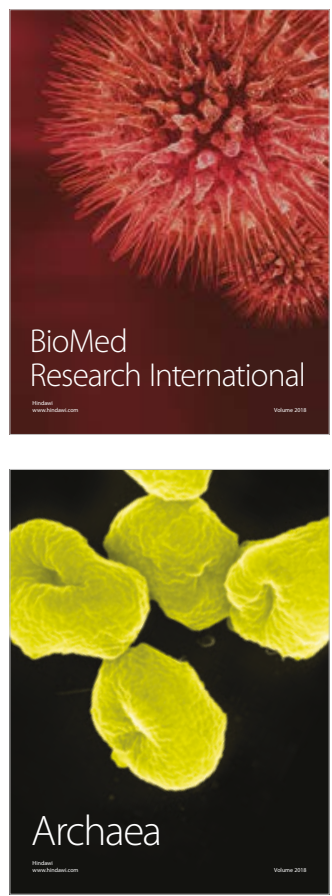